\title{
Impact of spiral separator geometrical parameters on the density separation of various fine-grained materials
}

\author{
Jan Szpyrka ${ }^{1, *}$, Tomasz Suponik ${ }^{2}$, and Marcin Lutyński ${ }^{1}$ \\ ${ }^{1}$ Silesian University of Technology, Faculty of Mining and Geology,44-100 Gliwice ul. Akademicka 2, Poland
}

\begin{abstract}
The study aims at the assessment of the impact of geometrical parameters of spiral separators on the efficiency of density separation of fine-grained materials. Experiments were carried out on three spiral separators: Krebs 2.85, Reichert LD-4 and Reichert LG-7. Three materials were used for the tests: raw coal, coal waste and mix of sand and magnetite as the model material. Results of raw coal and coal waste upgrading showed that density separation was most efficient in Reichert LD-4 spiral . This is due to the fact that this device had the highest amount of coils, height of sluice as well as was equipped with additional dense product collector and additional water sluice for transport water. The lower slope of sluice and larger height made separation even more efficient. Analysis of separation of model material, that is the mix of sand and magnetite, showed that in this case the existence of additional water sluice does not have an impact on product separation and best results were obtained in the Reichert LG-7 spiral separator. The shape and width of sluices did not have a significant impact on the separation process.
\end{abstract}

\section{Introduction}

Spiral separators are commonly used for the separation of fine-grained materials with the particle size not exceeding $3 \mathrm{~mm}$. The lower limit of particle size is $0,1 \mathrm{~mm}$ for the low density materials and $0,05 \mathrm{~mm}$ for heavy minerals $[1,2]$. The arrangement of material in accordance with its density in the sluice is determined by the liquid pressure which moves particles in the water stream [3]. As larger and heavier particles sink to the bottom of the sluice faster and experience more drag from the bottom, they travel slower, and so move towards the center of the spiral. Conversely, light particles stay towards the outside of the spiral, with the water, and quickly reach the bottom. Other forces also act on the particles and these are: gravity where direction is always constant for each particle and the force depends on the particle density; centrifugal force which results from the spiral movement of the water stream; friction which is the result of the slide of the particle on the surface of the sluice [4,5]. These forces act on the particles and lighter particles move with a higher speed whereas centrifugal force pushes particles to the outer side of the spiral [6]. The friction increases towards the inner side of the sluice whereas the centrifugal force decreases towards the inner side. Hence, dense particles tend to concentrate on the inner side of the sluice [7].

The efficiency of separation in the spiral separator depends on various factors such as feed parameters i.e. particle size distribution, density fraction and slurry density or geometrical parameters of spiral separator i.e. number of coils, angle and width of spiral as well as location of separating slots (fans) and number of heavy product collectors. Geometrical parameters have an impact on the precision of separation, yield of products and water stream movement in the sluice. Water stream in the sluice has varying height which increase towards the outer side of the sluice. The speed of the water stream changes similarly and the highest speed is at the outer edge where light particles are concentrated.

The purpose of this study was to assess the impact of geometrical parameters of three types of spiral separators (Krebs 2.85, Reichert LD-54, Reichert LG-7) on the efficiency of separation of various fine-grained materials. The practical application of this study was to select highly efficient spiral separator for the concentration of coal from coal waste slurries deposited in impoundments.

\section{Materials and methods}

Three types of materials with different density fractions were used in the study, these were: raw coal, coal waste from a dump and model material consisting of sand and magnetite mix. All three materials were separated in the spiral concentrator with the same slurry density of 300 $\mathrm{g} / \mathrm{dm}^{3}$.

Each separator is characterized by different geometrical parameters mainly number of spirals, slope of the sluice, width and shape of the sluice, diameter of the spiral, location of separating fans (slots) and number of heavy product collectors. These parameters are as follows:

- Krebs 2.85 separator: height $1750 \mathrm{~mm}$; spiral diameter $900 \mathrm{~mm}$; number of coils 2,85 ; sluice slope $15^{\circ}$; sluice width $280 \mathrm{~mm} \mathrm{-} \mathrm{including} \mathrm{additional} \mathrm{sluice} \mathrm{for}$

\footnotetext{
* Corresponding author: jan.szpyrka@polsl.pl
} 
transporting water of $70 \mathrm{~mm}$; shape of sluice - circular sector with flattened bottom, the outer part of the sluice is of $8 \mathrm{~cm}$ in height and bend $90^{\circ}$. Additional sluice for transporting water is the u-shape sluice $6,5 \mathrm{~cm}$ in depth; distance between separating cuts for heavy product, semiproduct and light product is $100,80,85 \mathrm{~mm}$ respectively; heavy product collectors - underflow at the bottom of spiral separator; spiral is made of plastic and covered with liners. There are two sluices with three coils each.

- Reichert LD-4 spiral: height $2500 \mathrm{~mm}$, spiral diameter $900 \mathrm{~mm}$; number of coils 6 ; sluice slope $15^{\circ}$; sluice width $280 \mathrm{~mm}$ - including additional sluice for transporting water of $60 \mathrm{~mm}$; shape of sluice - circular sector with flattened bottom; the outer part of the sluice is of $8,5 \mathrm{~cm}$ in height and bend $90^{\circ}$; additional sluice for transporting water is a flat shaped sluice $6 \mathrm{~cm}$ in depth; distance between separating cuts for heavy product, semi-product and light product is $100,80,80 \mathrm{~mm}$ respectively; heavy product collectors - underflow at the bottom of spiral separator and one additional collector in the middle of the spiral (third coil); spiral is made of plastic without liners. There are two sluices with six coils each.

- Reichert LG-7 spiral: height $2500 \mathrm{~mm}$, spiral diameter $580 \mathrm{~mm}$; number of coils 6 ; sluice slope $20^{\circ}$; sluice width $200 \mathrm{~mm}$; shape of sluice - circular sector with flattened bottom; the outer part of the sluice is of $6,5 \mathrm{~cm}$ in height and bend $90^{\circ}$; no additional sluice for transporting water; distance between separating cuts for heavy product, semiproduct and light product is $40,60,70 \mathrm{~mm}$ respectively; heavy product collectors - underflow at the bottom of spiral separator. This spiral separator, in comparison to the other two, is much narrower and the sluice slope is more inclined hence the flow speed of material and water is higher.

Table 1. Ash content in particle size fractions of raw coal and coal waste.

\begin{tabular}{|c|c|c|c|c|}
\hline \multirow{2}{*}{$\mathbf{\delta} . \mathbf{g} / \mathbf{c m}^{\mathbf{3}}$} & \multicolumn{2}{|c|}{ Raw coal } & \multicolumn{2}{c|}{ Coal waste } \\
\cline { 2 - 5 } & $\boldsymbol{\gamma} \mathbf{. \%}$ & $\boldsymbol{\lambda}_{\text {ash. } \mathbf{\%}}$ & $\boldsymbol{\gamma} \mathbf{\%}$ & $\boldsymbol{\lambda}_{\text {ash. }} \mathbf{\%}$ \\
\hline$<1.4$ & 79.48 & 3.08 & 53.56 & 7.46 \\
\hline $1.4-1.6$ & 5.28 & 22.04 & 5.28 & 27.91 \\
\hline $1.6-1.8$ & 3.03 & 37.27 & 11.58 & 70.29 \\
\hline$>1.8$ & 12.21 & 80.19 & 29.58 & 70.68 \\
\hline$\delta$ - density fraction; $\boldsymbol{\gamma}$ - yield; $\lambda$ ash - ash content \\
\hline
\end{tabular}

Table 2. Ash content in density fractions of raw coal and coal waste.

\begin{tabular}{|c|c|c|c|c|}
\hline \multirow{2}{*}{ Particle size. mm } & \multicolumn{2}{|c|}{ Raw coal } & \multicolumn{2}{c|}{ Coal waste } \\
\cline { 2 - 5 } & $\boldsymbol{\gamma . \%}$ & $\boldsymbol{\lambda}_{\text {ash. } \%}$ & $\boldsymbol{\gamma} \mathbf{\%}$ & $\boldsymbol{\lambda}_{\text {ash . \% }}$ \\
\hline$>3$ & 12.80 & 19.89 & 9.30 & 32.86 \\
\hline $3-2$ & 14.69 & 24.01 & 18.96 & 35.83 \\
\hline $2-1.5$ & 11.03 & 22.36 & 14.87 & 33.02 \\
\hline $1.5-1$ & 12.95 & 19.64 & 21.84 & 31.36 \\
\hline $1-0.5$ & 16.43 & 20.21 & 28.26 & 33.16 \\
\hline$<0.5$ & 32.10 & 21.77 & 6.78 & 49.13 \\
\hline Average & & 21.39 & & 34.31 \\
\hline$\gamma$ - yield; $\lambda_{\text {ash }}$ - ash content \\
\multicolumn{4}{|l|}{} \\
\hline
\end{tabular}

Both particle size and denisty analysis were carried out in accordance with national standards. Ash content was measured according to the polish standard PN-G-
04512:19809/A/1:2002. Magnetic separation was carried out on the Satmagan separator.

Ash content in each particle size fraction of raw coal and coal waste is presented in Table 1 whereas the ash content in denisty fraction of raw coal and coal waste is shown in Table 2.

Model material was created by mixing sand and magnetite in the mass ratio of $1: 4$. The particle size range of feed was $0-2 \mathrm{~mm}$. Average magnetite content in the feed of the model material was $24,28 \%$.

\section{Results and discussion}

Results of ash content analysis $\lambda_{\text {ash }}$ in separation products of raw coal, coal waste and model material (magnetite and sand mix) are shown in Tables 3, 4 and 5 respectively. In Table 3 the product of density separation of LG-7 separator is not shown since it was not possible to separate fine-grained raw coal in this device. High slope of sluice in LG-7 separator causes high flow speed of the slurry which results in slurry overflow from sluice and inefficient separation.

Initial assessment of density separation presented in Tables 3, 4 and 5 indicates that Krebs 2.85 and Reichert LD-4 separators are more efficient in upgrading raw coal and coal waste, whereas Reichert LD-7 is more efficient in upgrading sands and ores.

Detailed analysis of density separation results of raw coal shown in Table 3 revealed that this feed was upgraded more efficiently with the use of Reichert LD-4 separator. Ash content in concentrate of Krebs 2.85 separator was slightly lower, yet the yield of concentrate in Reichert LD-4 was much higher. Comparison of the two separators showed that tailings of the Reichert LD-4 have high concentration of ash and lower yield.

When comparing results of coal waste upgrading, the Reichert LG-7 spiral was the least efficient as well. Although yields of products were satisfactory (see Table 4) but the overall ash content in tailings and middlings was considerably high. Among other two separators Reichert LD-4 was more efficient in density separation of coal waste. The ash content in Krebs 2.85 was only slightly lower than in Reichert LD-4 yet the concentrate yield for the latter one was considerably higher. Overall, the efficiency of separation for the Reichert LD-4 was higher when considering the ash content and yields in middlings and tailings.

Particle size and ash content analysis of separation products of raw coal (Table 6) indicate that the low efficiency of Krebs 2.85 separator might be caused by the low number of coils. Because of that the residual time of particles feed in the separator is low and as a result particles do not separate resulting in high ash content in tailings. The fine particle size fraction of middlings from this separator had a low ash content indicating the process of agglomeration of fine particles with coarse ones and as a consequence - decrease in separation efficiency. Relatively high content of coal in tailings confirmed low separation efficiency which was caused be the short (height) of the spiral separator despite the fact that it is equipped with additional water sluice. 
Table 3. Ash content $\left(\lambda_{\text {ash }}\right)$ in products of raw coal upgrading in Krebs 2.85 and Reichert LD-4.

\begin{tabular}{|c|c|c|c|c|}
\hline \multirow{2}{*}{ Products } & \multicolumn{2}{|c|}{ Krebs $\mathbf{2 . 8 5}$} & \multicolumn{2}{c|}{ Reichert LD-4 } \\
\cline { 2 - 5 } & $\begin{array}{c}\boldsymbol{\gamma} \cdot \\
\mathbf{\%}\end{array}$ & $\boldsymbol{\lambda}_{\text {ash }} \mathbf{\%}$ & $\begin{array}{c}\boldsymbol{\gamma} \\
\mathbf{\%}\end{array}$ & $\begin{array}{c}\boldsymbol{\lambda}_{\text {ass. }} \\
\mathbf{\%}\end{array}$ \\
\hline Concentrate & 29.88 & 7.93 & 70.66 & 8.37 \\
\hline Middlings & 32.88 & 13.65 & 9.69 & 20.66 \\
\hline Tailings & 37.24 & 32.98 & 19.65 & 55.02 \\
\hline
\end{tabular}

Table 4. Ash content ( $\left.\lambda_{\text {ash }}\right)$ in products of coal waste upgrading in Krebs 2.85, Reichert LD-4 and Reichert LG-7.

\begin{tabular}{|c|c|c|c|c|c|c|}
\hline \multirow{2}{*}{ Products } & \multicolumn{2}{|c|}{ Krebs 2.85} & \multicolumn{2}{|c|}{$\begin{array}{c}\text { Reichert LD- } \\
4\end{array}$} & \multicolumn{2}{|c|}{$\begin{array}{c}\text { Reichert LG- } \\
7\end{array}$} \\
\hline & $\begin{array}{l}\gamma . \\
\%\end{array}$ & $\begin{array}{c}\lambda_{\text {ash }} \\
\%\end{array}$ & $\begin{array}{l}\gamma . \\
\% \\
\%\end{array}$ & $\begin{array}{c}\lambda_{\text {ash }} \\
\%\end{array}$ & $\begin{array}{l}\% \\
\%\end{array}$ & $\begin{array}{c}\lambda_{\text {ash }} \\
\%\end{array}$ \\
\hline Concentrate & 24.90 & 13.88 & 48.86 & 14.91 & 81.20 & 30.56 \\
\hline Middlings & 29.10 & 24.27 & 21.65 & 31.58 & 16.06 & 50.35 \\
\hline Tailings & 46.00 & 47.89 & 29.49 & 53.61 & 2.74 & 64.21 \\
\hline
\end{tabular}

Table 5. Magnetite content $\left(\lambda_{\mathrm{m}}\right)$ in products of model material (magnetite+sand) in Krebs 2.85, Reichert LD-4 and Reichert LG-7 separators.

\begin{tabular}{|c|c|c|c|c|c|c|}
\hline \multirow{2}{*}{ Products } & \multicolumn{2}{|c|}{ Krebs $\mathbf{2 . 8 5}$} & \multicolumn{2}{|c|}{$\begin{array}{c}\text { Reichert LD- } \\
\mathbf{4}\end{array}$} & \multicolumn{2}{|c|}{$\begin{array}{c}\text { Reichert } \\
\text { LG-7 }\end{array}$} \\
\cline { 2 - 7 } & $\begin{array}{c}\boldsymbol{\gamma} \mathbf{\%} \\
\mathbf{\%}\end{array}$ & $\begin{array}{c}\boldsymbol{\lambda}_{\mathbf{m}} \cdot \\
\mathbf{\%}\end{array}$ & $\begin{array}{c}\gamma \cdot \\
\mathbf{\%}\end{array}$ & $\begin{array}{c}\boldsymbol{\lambda}_{\mathbf{m}} \cdot \\
\mathbf{\%}\end{array}$ & $\begin{array}{c}\gamma \cdot \\
\mathbf{\%}\end{array}$ & $\begin{array}{c}\boldsymbol{\lambda}_{\mathbf{m}} \\
\mathbf{\%}\end{array}$ \\
\hline $\begin{array}{c}\text { Tailings } \\
\text { (light } \\
\text { product) }\end{array}$ & 10.37 & 52.70 & 2.50 & 86.07 & 48.45 & 8.33 \\
\hline Middlings & 11.96 & 9.70 & 1.11 & 60.70 & 41.87 & 31.00 \\
\hline $\begin{array}{c}\text { Concentrate } \\
\text { (heavy } \\
\text { product) }\end{array}$ & 77.67 & 20.93 & 96.39 & 19.63 & 9.39 & 81.07 \\
\hline
\end{tabular}

More detailed analysis of products from the Reichert LD-4 separator (Table 6) indicate that high ash content in the concentrate was caused by the inflow of light products above $3 \mathrm{~mm}$ in size which should have been separated in the additional sluice. These particles were "thrown out" to the outer side of the sluice. Middlings and tailings had slightly better properties than the ones obtained in the Krebs 2.85 separator which could be attributed to the higher number of coils in the latter one.

High ash content in the concentrate of Krebs 2.85 separator (table 7) was also caused by the passing of coarse grains $(>2 \mathrm{~mm})$ with high ash content into this product. These particles should have stayed in the additional water sluice where separation should have taken place. Low ash content in dense particle size fractions showed that separation was insufficient due to the low number of coils. Additional transport water (sluice) was not able to separate coal from the dense fraction (tailings).

Relatively high ash content in the concentrate from the coal waste of the Reichert LD-4 spiral (Table 7) can be attributed to the content both of fine and coarse particles. The latter ones were pushed out to the outer side of the sluice. When comparing particle sizes and density fraction of dense products from this separator with Krebs 2.85 it is evident that better separation efficiency is obtained in spiral separators with higher number of coils.
Table 6. Ash content $\left(\lambda_{\text {ash }}\right)$ in particle size fractions of raw coal upgrading in Krebs 2.85 and Reichert LD-4 separators.

\begin{tabular}{|c|c|c|c|c|c|c|}
\hline \multirow{3}{*}{ 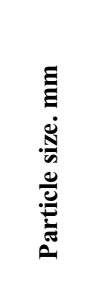 } & \multicolumn{6}{|c|}{ Ash content $\lambda_{\text {ash }}(\%)$ in particle size fractions } \\
\hline & \multicolumn{3}{|c|}{ Krebs 2.85} & \multicolumn{3}{|c|}{ Reichert LD-4 } \\
\hline & 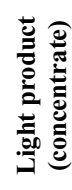 & 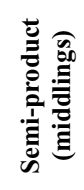 & 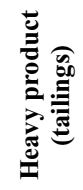 & 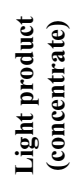 & 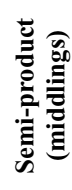 & 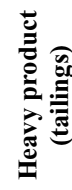 \\
\hline$>3$ & 6.08 & 25.46 & 39.39 & 17.03 & 32.96 & 73.66 \\
\hline $3-2$ & 7.01 & 20.72 & 44.86 & 8.78 & 40.93 & 74.51 \\
\hline $2-1.5$ & 4.26 & 14.80 & 41.82 & 9.84 & 43.07 & 73.63 \\
\hline $1.5-1$ & 5.33 & 9.14 & 36.00 & 6.49 & 31.61 & 68.41 \\
\hline $1-0.5$ & 3.58 & 14.96 & 31.48 & 4.55 & 11.69 & 52.99 \\
\hline$<0.5$ & $\begin{array}{c}14.3 \\
9\end{array}$ & 6.52 & 25.77 & 9.03 & 9.88 & 40.33 \\
\hline
\end{tabular}

Table 7. Ash content $\left(\lambda_{\text {ash }}\right)$ in particle size fractions of coal waste upgrading in Krebs 2.85 and Reichert LD-4 separators.

\begin{tabular}{|c|c|c|c|c|c|c|}
\hline \multirow{3}{*}{ 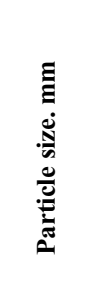 } & \multicolumn{6}{|c|}{ Ash content $\lambda_{\text {ash }}(\%)$ in particle size fractions } \\
\hline & \multicolumn{3}{|c|}{ Krebs 2.85} & \multicolumn{3}{|c|}{ Reichert LD-4 } \\
\hline & 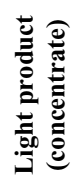 & 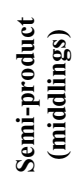 & 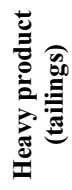 & 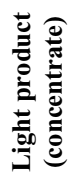 & 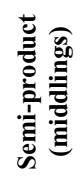 & 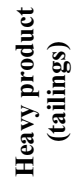 \\
\hline$>3$ & 19.53 & 36.85 & 43.06 & 20.96 & 42.25 & 52.15 \\
\hline $3-2$ & 18.15 & 35.21 & 55.52 & 19.16 & 49.33 & 58.86 \\
\hline $2-1.5$ & 11.46 & 29.81 & 50.70 & 13.98 & 42.91 & 58.19 \\
\hline $1.5-1$ & 7.45 & 20.68 & 46.89 & 10.32 & 32.6 & 55.84 \\
\hline $1-0.5$ & 6.54 & 13.52 & 44.81 & 8.42 & 18.64 & 49.05 \\
\hline$<0.5$ & 18.30 & 21.30 & 54.25 & 34.99 & 28.39 & 59.65 \\
\hline
\end{tabular}

Results of separation of sand and magnetite mixture (Table 5) indicate that the most efficient separation was in Reichert LG-7. Highest yield of light product with low content of magnetite and lowest yield of the heavy one with high content of magnetite were obtained in this separator. In the other two separators (Krebs 2.85 and Reichert LD-4) results were not as expected, in both cases the highest content of magnetite was observed in the light product (Table 5). Also the yields of products showed inefficient separation process.

\section{Results and conclusions}

For the purpose of coal upgrading Reichert LD-4 spiral is the best choice. This is due to the following reasons:

- higher number of coils and height causes longer residual time of particles in the sluice and as a consequence more accurate density partition,

- existence of additional dense product collector which is located in the middle of the separator height and receives tailings separated in the first phase of separation,

- existence of additional water sluice which increases efficiency of separation of a "clean" heavy product (with high ash content) minimizing at the same time its yield. Lack of this sluice in Reichert LG-7 spiral causes contamination of concentrate grains with light product 
grains which as a consequence increases ash content in concentrate (table 4),

- Lower sluice slope inclination which causes lower flow rates hence more efficient separation,

- Larger sluice width - the LG-7 separator sluice width is $200 \mathrm{~mm}$ and free separation of material is reduced which results in the mixing of products.

One significant observation was made during the course of experiments - the location of "cuts" does not have a major impact on the efficiency of separation. Location of cuts (slots) was almost the same in case of Krebs 2.85 and Reichert LD-4 i.e. the largest gap was for the tailings $-100 \mathrm{~mm}$, smaller for the middlings and concentrate $-80 \mathrm{~mm}$ and $85 \mathrm{~mm}$, for Krebs 2.85 the gaps were 80 and $80 \mathrm{~mm}$ respectively. Despite of it, analysis of products showed some large differences.

The shape of sluice did not differ significantly in the separators and did not have a substantial impact on the process of separation.

Analysis of separation of model material, that is the mix of sand and magnetite, showed that in this case the existence of additional water sluice does not have an impact on product separation. Best results were obtained in the Reichert LG-7 spiral separator which is not equipped with the additional sluice. In case of Krebs 2.85 and Reichert LD-4 a small amount of magnetite was found in the additional sluice. The number of coils did not have an impact on the efficiency of separation which was proven by the fact that the least favorable results were obtained in the Reichert LD-4 spiral with the same number of coils as the Reichert LG-7. The shape and width of sluices did not have a significant impact on the separation process.

\section{References}

1. A. B. Holland-Batt, P.N. Holtham, Miner. Eng., 4, (1991)

2. R. G. Richards, D. M. MacHunter, P. J. Gates, M. K. Palmer, Miner. Eng., 13, (2000)

3. Y. M. Stokes, Computing Flow in a Spiral Particle Separator (14th Australasian Fluid Mechanics Conference Adelaide University, Adelaide, 2001)

4. W. Blaschke, S. Blaschke. Technika wzbogacania grawitacyjnego. Wzbogacalniki strumieniowe (Wyd. Inst. Gosp. Sur. Min. i Energią PAN, 1999)

5. J. Drzymała. Podstawy mineralurgii, (Oficyna Wydawnicza Politechniki Wrocławskiej, Wrocław, 2009)

6. Z. Blaschke, Inżynieria Mineralna, 1(2), 33-36, (2000)

7. D. K. Henderson, D. M. MacHunter, Mineral Technologies, (2003) 